\title{
La historia de la traducción como tarea de investigación de las letras costarricenses ${ }^{1}$
}

\author{
Miguel Ángel Vega Cernuda²
} Universidad de Alicante, España

\section{RESUMEN}

Se pone de relieve la importancia histórica y pertinencia cultural del desarrollo de la traducción en Costa Rica, en particular de su literatura. Se exploran algunos antecedentes y referencias al asunto, se señalan algunos déficits significativos y se plantean cinco propuestas teóricas: existe una dependencia de las literaturas nacionales de su traducción; la traducción debe formar parte de las historias literarias nacionales; se debe explicitar la relación entre la historiografía política y social y la traducción; se debe considerar el patrimonio literario como parte de la identidad cultural; por último, la traducción debe verse como parte de la memoria de la humanidad y, por tanto, su relevante valor cultural.

\section{Abstract}

Emphasis is given to the historical importance and cultural pertinence of the development of translation in Costa Rica, and of its literature in particular. Some background and references are provided, and significant gaps are addressed, along with five theoretical proposals: National literatures depend on being translated; translation should be part of national literary histories;

i Las presentes páginas fueron leídas en el I Congreso Internacional de Linguiística Aplicada, celebrado en octubre de 2007, en la ciudad de Heredia, en el Campus Omar Dengo de la Universidad Nacional de Costa Rica.

2 Correo electrónico: miguel.vega@ua.es 
the relation between political and social historiography and translation should be explicit; literary heritage should be part of cultural identity; and translation should be seen as part of the memory of humanity, and in turn its relevant cultural value recognized.

Palabras clave: historia de la cultura, traducción, historiografía literaria, historia de la traducción, literatura costarricense.

Keywords: history of culture, translation, literary historiography, history of translation, Costa Rican literature.

El que les habla no podría calibrar si, como dijo Miguel Obregón Lizano, Costa Rica «destaca más por la belleza de sus paisajes que por su cultura», pero resulta evidente que Costa Rica manifiesta, justa o injustamente, un déficit de imagen cultural cuando en el catálogo Opale de la Biblioteca Nacional de Francia no aparece, por ejemplo, ninguna referencia a Max Jiménez. Su Quijongo, aparecido en Madrid en 1933, no existe en los registros de la BNF. Ni siquiera la traducción francesa de Gleba, publicada en 1929 en París, está ese archivo de la memoria colectiva que es la Biblioteca Nacional de París. De Alberto F. Cañas no existen en la actualidad más que una veintena de registros en los catálogos alemanes, todos ellos en español. (La Segua, por ejemplo). Lo cual evidencia que, mientras este autor no se traduzca, estará condenado a la inexistencia en la memoria de la humanidad. Aunque hoy en día tenemos una conciencia hipertrofiada en lo que a conservación del patrimonio cultural humano se refiere, el libro, y más si se trata de uno traducido, no encuentra un interés que salvaguarde su supervivencia. Hoy en día despierta mayor interés una colección de mariposas que un libro en vías de extinción. Porque, dicho sea de paso, los libros también se mueren. Y a este respecto, el caso de la literatura costarricense es especialmente grave.

Por otra parte, una análisis de la producción bibliográfica nacional pone de manifiesto que en la misma predomina la producción primaria; es decir, la creación y los estudios sobre esa producción primaria (ensayos de cultura étnica o antropológica, etc.) faltando casi 
por completo textos secundarios, de recepción y fenómenos semejantes que tienen en la traducción uno de sus ejes. Son escasos los títulos a ejemplo del de Virginia Borloz («Flaubert en la literatura costarricense»). Hace ya años, en 1958, se celebró en Costa Rica el Congreso Internacional de Americanistas. En sus actas se recogía un trabajo de Guillermo Malavassi que tematizaba la «Presencia de Unamuno en Costa Rica». El contenido y estructuración del trabajo respondía a lo que podrían ser las bases para una historia de la ilustración intelectual del país. Y en este sentido deberían multiplicarse los trabajos de recepción crítica y traductográfica de un Shakespeare, un Goethe, un Faulkner o un T.S. Elliot, por ejemplo, en Costa Rica. Pues, en ef ecto, el panorama intelectual de la nación ha dependido precisamente de las obras de estos autores han leído sus grandes o incluso medianos hombres. Sin embargo, de las 680 entradas bibliográficas que el gran catálogo virtual alemán GVB (Gemeinsamer Bibliotheksverbund) contiene sobre los más diversos temas de la vida nacional y que se presentan bajo el epígrafe "costarricense" (política, derecho, arte, narrativa, indígenas, historiografía, gastronomía, hostelería, seguridad ciudadana, derecho electoral, el trapiche costarricense, el anglicismo costarricense, la carreta costarricense etc., son temas que pasan por el elenco con títulos tan variopintos y específicos como Entre el comal y la olla: fundamentos de gastronomía costarricense o Haciendo patria con el paisaje costarricense: la consolidación de un arte nacional en la década de 1930) no hay nada que remotamente se aproxime al tema de la traducción literaria, que es la que aquí, sobre todo, focalizamos. Podemos aceptar como hecho indiscutible que el epígrafe traducción costarricense o traducción en Costa Rica no existe como asunto o materia de las clasificaciones bibliográficas. Por eso se agradece que, por ejemplo, la obra de Carlos Francisco Monge acerca de la vanguardia costarricense (El vanguardismo literario en Costa Rica) $)^{3}$, se haga, al menos ocasionalmente, alusión a algunas

3 Carlos Francisco Monge, El vanguardismo literario en Costa Rica (Heredia: Editorial Universidad Nacional, 2005). 
traducciones que pudieron ejercer su influencia en el decurso literario de la nación: «Abundan [en las nuevas revistas de los años 20] noticias sobre la actualidad política internacional, comentarios... incluidas traducciones y una gran atención a la literatura contemporánea, no solo la escrita en español» (pag. 23). La actividad de la Editorial Costa Rica, con un elenco largo de autores y títulos (Cañas, Chase, Fallas, Rossi, etc.) no ha recogido, en la medida en que he podido comprobarlo a través del análisis online de sus catálogos, un solo título de traducción. A la hora de reseñar la historia de la literatura nacional, todos son títulos nacionales, es decir, originales en español costarricense. En el interesante estudio acerca de la narrativa costarricense «Historia y narrativa en Costa Rica», en la revista Istmo, firmado por Álvaro Quesada Soto, se hace un interesante esbozo de los contextos del tema, que se sitúa en medio de las conmociones y determinaciones socio-políticas del país (Guerra Civil, neo-liberalismo, etc.), si bien con exclusión absoluta de toda referencia a una causalidad que le hubiera advenido a esta narrativa por vía literaria extranjera. Se supone que las traducciones que se hicieran, publicaran o leyeran en el país no fueron causas determinantes de esa narrativa, lo que desde el punto de vista de la fenomenología literaria es difícilmente aceptable. Si la exposición del Club Alemán del año 32 sobre el Expresionismo Alemán se aduce como factor de evolución estética en el ideario de Amighetti, justo será, al menos, suponer que las obras traducidas de la vanguardia europea y mundial pudieron influir en el desarrollo de la vanguardia nacional. El célebre trabajo de Abelardo Bonilla Historia de la Literatura Costarricense ${ }^{4}$ tiene escasas alusiones a versiones y en todo caso no reserva ningún epígrafe al género. En la historia de un país multilingüe como fue este (con lenguas como el bribri, el cabécar, etc.) se hace caso omiso de la labor de comunicación interlingüística que tuvo que mediar en la convivencia nacional. Así

$4 \quad$ Abelardo Bonilla, Historia de la Literatura Costarricense. 2a ${ }^{\mathrm{a}}$ ed. (San José: Editorial Costa Rica, 1967). 
en Vida cotidiana en la Costa Rica del siglo XIX, de González Ortega ${ }^{5}$ se habla del asentamiento de la vida religiosa en el pueblo sin mencionar la posible actividad traductora de la iglesia frente a los indígenas.

Ante este panorama, me atrevería a lanzarles una pregunta, más allá de la retórica: ¿es que, en cuanto grupo profesional, el traductor costarricense se resigna a no tener historia, a no tener conciencia del gremio al que pertenecen, y sin el cual quizás su país no sería lo que es? Las «traducciones ticas» a las que hacía referencia José Figueres Ferrer, en un estudio misceláneo acerca de Joaquín Gutiérrez, ¿no merecen un estudio de su función social y de su valor literario y nacional? Bien es verdad que este fundador de la II República, establecería una ecuación - peligrosa por denigratoria- de la labor del traductor cuando, a la hora de referirse a las traducciones de Gutiérrez, titulaba su aportación: Traducciones y otras vagabunderías (en Rodolfo Arias Formoso, Retrato de Joaquín Gutiérrez $z^{6}$ ). ¿Es que la traducción es una vagabundería? Quizás no haya que darle mayor importancia a la fórmula, pero en todo caso es la reacción típica de un político ante unas letras que paulatinamente se le hacen más extrañas.

No creo que pueda discutirse que sería interesante que la editorial que suministra parcialmente las señas de identidad literaria a la nación, pusiera a disposición de su público las traducciones canónicas, nacionales o extranjeras, por ejemplo, del Hamlet de Shakespeare, el Fausto de Goethe o la Divina Comedia del Dante. Mientras no se llegue a eso, la obligación de una ilustración nacional estará posiblemente limitada a los académicos y escritores, pero no llegará a las clases populares. Y en todo caso esa ilustración foránea que llega a Costa Rica igual que a cualquier país debería investigarse lo mismo que la música criolla. Tiene Carlos Francisco Monge más razón que un

5 Alfonso González Ortega, Vida cotidiana en la Costa Rica del siglo xIX (San José: Editorial de la Universidad de Costa Rica, 1997).

6 Rodolfo Arias Formoso, Retrato de Joaquín Gutiérrez (San José: Editorial de la Universidad de Costa Rica, 2002). 
santo padre cuando, en la obra antes mencionada, afirma que «el desarrollo de una literatura no es solo un asunto estrictamente sociológico». En un país en el que este factor ha gozado de un primer plano en la actualidad histórica, se corre el peligro de creerlo. La situación traductora de Costa Rica, un país que en el registro de traducciones que realiza la UNESCO, el Index Translationum, da un panorama bastante escaso de actividad, contrasta con la enorme actividad intelectual que el país registra. Y con ello ya estoy mencionando ese déficit de imagen que debería corregirse. El conjunto de habitantes de la galaxia hispanohablante contribuye a enriquecer unas maneras de ver el mundo y de expresarlas; lo cual resulta valor cultural irrenunciable y que se tiene la obligación de difundir... a través de la traducción. Las traducciones de un Valera, de un Amado Nervo, de un Francisco Jiménez (Popol Vuh) son importaciones que, al tiempo que acrecientan nuestro acervo cultural y lingüístico, proyectan versiones diferentes sobre las producciones foráneas que nos universalizan en la medida en que las hacemos propias. Las traducciones que, mejores y peores, se realizaron de los clásicos de la economía y la política en México en los años 40-70 (desde el Colegio de México, en parte labor de los emigrados españoles, como Altolaguirre, Roces, Gaos) o, también en los años 40-70, de la literatura europea en Argentina (en unos momentos en los que todavía no disponíamos de versiones alemanas de Rilke o Kafka) son episodios que han dado consistencia al conjunto de nuestros pueblos. No es pensable que en ese amplio contexto cultural falte la visión costarricense.

Frente a este estado de la «cosa traductiva», quisiera hacerles unas propuestas teóricas que no por estar próximas a la verdad de Perogrullo son menos verdad. Son cuatro propuestas de fenomenología literaria y cultural que se podrían discutir o incluso negar, pero que pretendo hacer valer como materia de reflexión en este Congreso y que quiero que sirvan de motivación y punto de partida a un entusiasta trabajo de investigación común, a saber, el de la historia de la traducción de Costa Rica, en el que nos gustaría empeñarnos, pues la causa lo merece. 
1. Es mi primera propuesta la de la dependencia de cada literatura nacional de la traducción. Roma empezó a tener conciencia de su vocación literaria cuando Livio Andrónico tradujo la Odisea de Homero; los germanos empezaron a desarrollar una literatura artística con las traducciones de los textos de la liturgia cristiana; con la traducción de la Biblia de Lutero se inicia el alemán moderno; el árabe culto se nutre de las traducciones que se realizaron en los siglos IX y $x$ en la Casa de la Sabiduría de Bagdad y el despertar de la cultura renacentista fue la llamada Escuela de Toledo. La cultura eslava se formó gracias a las traducciones de Cirilo y Metodio, padres de su alfabeto. Podíamos seguir mencionando los efectos de la traducción en el despertar poético y cultural de las naciones pero basten esos ejemplos.

2. Es la segunda una propuesta de historiografía literaria: la historia de la literatura hasta el presente está mal escrita, ya que ha omitido una parte fundamental de las bases escritas de un grupo humano: los transtextos, aquellos textos emigrados que aportan a una cultura el fermento de la alteridad. Cuando escribimos la historia del Siglo de Oro español sin atender a las traducciones de los clásicos (Plutarco, por ejemplo) o de los modernos italianos (las obras de Petrarca) estamos dejando incompleto la documentación del cuadro cultural hispano del XVI. La traducción es el género original de toda literatura nacional. A pesar de eso, basta con abrir cualquier exposición de la historia de la literatura de un país o de una lengua para comprobar que uno de los factores más importantes de transmisión de la formación y del gusto literario, la traducción, brilla por su ausencia tanto en el proceso de creación poética de una lengua como en el resultado. Cualquiera que pretenda ingresar en la esencia del Siglo de Oro español, siglo de oro común a las naciones hispanas, pues a él contribuyeron, por ejemplo y entre otros una Juana Inés de la Cruz, un Antonio Alarcón o un Garcilaso, el Inca, tendrá que tener en cuenta de manera nuclear la presencia que en su entramado intelectual y literario 
desempeñaron, por ejemplo, las traducciones de Petrarca al español, que dieron lugar al petrarquismo de Garcilaso de la Vega, el tío, las traducciones de Erasmo, que dieron lugar al erasmismo español e incluso americano, o la traducción del Gracilazo, el sobrino inca, que determinó el platonismo español o las traducciones de los escritores piadosos alemanes que influyeron decisivamente en la mística española. Muy pocos son los manuales de historiografía que contemplan la traducción como un factor determinantes. Los trabajos de Alborg ${ }^{7}$ o de Valbuena $\mathrm{Prat}^{8}$ son suficientes para comprobarlo y las excepciones a esta regla son pocas. Los trabajos al respecto de Costa Rica van también en este sentido, como, por ejemplo, el de Abelardo Bonilla ${ }^{9}$.

Permítanme un ejemplo de lo que podía ser una ejemplar historia de la cultura y la literatura desde el punto de vista de la traducción. El erudito mexicano Antonio Alatorre, autor de una interesante crónica del castellano, (Los 1001 años de la lengua española ${ }^{10}$ ) brillante excepción a esa ignorancia de la traducción, en el extenso cuadro historiográfico que traza de nuestra lengua, está siempre atento a lo largo de su estudio a la causalidad que en el estado cultural del español ha tenido la traducción. Así, cuando hace mención de la presencia de Erasmo y de los humanistas a través de la versión de sus obras en las Indias:

«En lası Indias (...) la cultura que se fue implantando estaba hecha de la misma sustancia que en la metrópoli. Es verdad que hacia 1550 las únicas ciudades que podían llamarse centros de cultura eran México y Lima, y tal vez Santo Domingo (la primera que tuvo universidad). Pero, proporcionalmente, los ideales del Renacimiento y del humanismo penetraron en América en la misma medida que en España. Fernández de Oviedo,

\footnotetext{
Juan Luis Alborg, Historia de la literatura española (Madrid: Editorial Gredos, 1980). Angel Valbuena Prat, Historia de la Literatura Española (Barcelona : Gustavo Gili, 1946). Bonilla, Historia de la Literatura Costarricense.

Antonio Alatorre, Los 1001 años de la lengua española (México: Bancomer, 1979).
} 
imbuido de italianismo y lector de Erasmo, es en Santo Domingo uno de los españoles más civilizados de su tiempo, y su Historia uno de los monumentos del humanismo, entendido éste en su sentido más amplio y generoso. Diego Méndez, «el de la Canoa», compañero de Colón en su último viaje y vecino también de Santo Domingo, es famoso por el testamento(1536) en que dejó a sus hijos su biblioteca, formada por sólo diez libros, cinco de los cuales eran traducciones de Erasmo. Fray Juan de Zumárraga, primer obispo de México, reprodujo escritos de Erasmo y del erasmista Constantino Ponce de la Fuente. (Años después, este doctor Constantino fue encarcelado bajo acusación de luteranismo.) La Utopía del inglés Tomás Moro, amigo de Erasmo, tuvo innumerables lectores, pero ninguno tan extraordinario como Vasco de Quiroga, que quiso hacer realidad, en tierras de Michoacán, los ideales de justicia de ese libro revolucionario. Y Francisco Cervantes de Salazar, discípulo del erasmista Alejo Vanegas, no sólo tradujo a Juan Luis Vives, otro gran amigo de Erasmo sino que, a imitación suya, compuso unos Diálogos latinos impresos en México (1554), tres de ellos acerca de México y de su universidad, aún reciente pero ya activa» ${ }^{11}$.

3. La tercera proposición se referiría a la historiografía política y social: efectivamente, tampoco en ésta encuentra acomodo la traducción y parece como si se considerara que todas las relaciones internacionales se hubieran desarrollado en un único idioma prebabélico. A cualquiera que lea una historia de la Primera Guerra Mundial, el desarrollo de los hechos se le presentará como una sucesión de datos en los que al parecer la diferencia lingüística no hubiera desempeñado ningún papel. Sabido es, por ejemplo, que en la firma del armisticio de Compiègne hubo que improvisar una interpretación echando

11 En el caso de Alatorre nos encontramos con un ejemplo de cómo se debería escribir, desde la traducción, la historia literaria y la historia de una lengua. 
mano de uno de los mandatarios alemanes que hablaba francés, hecho éste que no suele figurar en las historias; menos se piensa que esto hubiera podido influir en el transcurso de las negociaciones de la capitulación o Armistice. Solo los historiadores de Indias, venidos de una koine más o menos perfecta por la todavía vigencia general del latín entre la clase culta, han vivenciado el estado complejísimo de comunicación que reinaba en el Nuevo Mundo y han sido conscientes del hecho interlingüístico. Por eso, es el mismísimo Almirante, quien en su diario apunta, ya el mismo día del descubrimiento, la necesidad de enseñar el español a algunos indígenas con el objeto de superar las barreras idiomáticas. Intentar rastrear la presencia histórica de los traductores en la historia del país es misión imposible, al menos para los que no disponemos de los medios de investigación que puedan darse en el país. La consulta online de fuentes produce escasos resultados. Desconozco si la actividad traductora en Costa Rica ha sido lo suficientemente intensa, pero en todo caso creo que es obligación nacional sacar a la luz las aportaciones que en este sentido haya hecho el país. La irrupción de la caficultura y del banano por obra y gracia de grandes empresas extranjeras tuvo que haber producido documentos que posiblemente hicieron intervenir a más de un traductor. Todavía Fallas en su Mamita Yunai alude al salpicado de topónimos ingleses a lo largo del trayecto de ferrocarril que le lleva a Puerto Limón. La historia general debe ayudarse de la historia de la traducción y viceversa. Crear compartimentos estancos es hacer anatomía y no fisiología. Es decir, es matar al bicho para estudiarlo, no mantenerlo en vida para comprobar su comportamiento.

4. La cuarta propuesta transciende lo estrictamente traductológico y va a lo cultural: el «patrimonio literario» de un grupo humano constituye una gran parte de su identidad cultural: para él se ha escrito y a él caracteriza. Naturalmente al conjunto de hablantes del español nos caracteriza el acervo literario común que poseemos de manera más o menos indirecta. Las Concherías de Aquileo J. Echevarría nos 
caracterizan y nos expresan a todos los hispanos en la medida en que a través del lenguaje llevan implícitas una visión del mundo más o menos común. Más allá de esto, esas «concherías» expresan y hacen especial referencia al campesino hispano y más en concreto al campesino costarricense. De ahí la perentoria necesidad de dar a conocer lo propio a través de la traducción, que es un vehículo importante de trasmisión de la propia imagen.

5. Un quinto apunte o consideración nos lleva a la fenomenología de la sociología cultural. La traducción literaria en cuanto documento en el soporte, relativamente consistente, del libro, forma parte de la común memoria de la humanidad. Documentos culturales, como lo son también las pinturas de nuestros museos o las monedas de las colecciones numismáticas, las traducciones sirven de testigo al proceso por el cual un producto condenado a la «localidad» se trasforma en un bien de consumo cultural «internacional». Las mencionadas Concherías estarán condenadas a no ver otros horizontes mientras atrevidos editores y traductores no se atrevan a importar ese espíritu del campesinado de las altas tierras costarricenses. Este contribuirá al panópticum de la cultura humana que gracias a él se vería más completa, más real. La traducción, en efecto, tiene una enorme capacidad de explicitación u ocultamiento, a través de «capturas» o «pérdidas» de los valores identitarios del «original» (bien sea colectivo - lengua, pueblo que la habla y crea, etc.— , bien sea individual - estilo, autor que lo fija, etc_-) que entrega u oculta a la vista del grupo humano para el que traduce. Scripta manent decían los clásicos: las traducciones serán huellas testigo del paso de las comunidades por la historia. Las traducciones pondrán de manifiesto lo que un grupo humano en una época concreta ha leído o ha podido leer.

De todo ello se sigue que la importancia del estudio de la traducción para Costa Rica es una cuestión de identidad nacional y que la investigación de aquella redunda en un mejor conocimiento de esta: 
Investigación de la exotraducción, porque con ella se da a conocer al exterior, e investigación de la endotraducción, pues gracias a ella el país ha incorporado e incorpora al patrimonio nacional nueva riqueza cultural. ¿Cuál es la situación al respecto en Costa Rica? Ustedes dirán, pero ustedes, en su calidad de miembros de la única especialidad de traducción en la Universidad Costarricense, están llamados a enriquecer el patrimonio cultural del país y con ello el de las letras hispanas... traduciendo e investigando la traducción. Unas traducciones hechas por costarricenses y quizás para costarricenses enriquecerían la multiforme expresión hispana. El que haya unas letras propias, nacionales, exige el complemento natural del "género" traducción. Vayan nuestras palabras de aliento para que traduzcan, para que hagan traducir y para que estudien la traducción.

La existencia de esas letras nacionales en el extranjero exige igualmente la correspondiente representación en la literatura mundial a través de la traducción, a través de la llamada exotraducción en el país. Tampoco en este apartado Costa Rica ha obtenido lo que se merece. Son muy escasos los autores que han logrado traspasar las fronteras, no por falta de méritos sino tal vez por falta de interés o por excesiva modestia, por el «tiquismo» llevado al extremo. Fallas, Gutiérrez, Rossi, por ejemplo, son autores que han accedido a la versión de sus obras en varias lenguas pero la literatura nacional que se ha hecho patrimonio internacional, de la literatura mundial es más bien escasa ¿Quiere decir esto que no hay autores costarricense que merecerían pasar a la que Goethe llamaba «literatura universal»? En absoluto. Lo cierto es que un conjunto de factores históricos quizás haya podido contribuir a un cierto déficit. De ellos me interesa destacar:

- en primer lugar, la juventud histórica de una país joven, ya que en su identidad nacional tiene escasamente dos siglos;

- la revuelta y agitada historia del país;

- la focalización de los intereses nacionales en asuntos sociales (ecología, turismo, etc.). 
Estos y muchos más son posibles factores del escaso fomento de la traducción de la propia literatura, que en todo caso habría que estudiar. No deja de resultar chocante que, por ejemplo, la música costarricense, a través del Teatro Nacional de San José, haya logrado una mayor internacionalización que su literatura.

Dicho eso y para finalizar, ¿cuáles serían las tareas y los métodos de la historia de la traducción? Para determinar y justipreciar tanto la esencia como el valor de la misma tendremos que considerar tanto lo que "esencialmente" es como lo que "históricamente" ha sido. Los análisis esencialistas tienden a la obtención de una traducción idealmente buena u óptima. Sobre esta base se han desarrollado escuelas tales como la de París, con su modelo interpretativo, la de Heidelberg, con su modelo del escopos o del encargo, etc. Pero además de buena o mala, adecuada o inadecuada - juicios finales de todo análisis esencialista - la traducción es o ha podido ser operativa o ineficaz, inmanente o trascendente, creadora de realidad estética o social, etc.; ha sido un medio que ha determinado el comportamiento y las actitudes mutuos de las naciones y los pueblos; es un hecho económico con una diacronía específica, que debe incluir el binomio fundamental de la economía, a saber, el de la of erta y la demanda y en cuya base hay que poner la actividad de un operario intelectual que, incluso en los casos de Jerónimo de Estridón o de Martín Lutero, entregados a la traducción sacra, depende de los «posibles» que esa traducción le proporcione para sobrevivir.

Todos estos son factores que determinan la existencia de la traducción como realidad social y a todos ellos debe atender una historia de la traducción que haga justicia al papel de ésta en la sociedad a lo largo del tiempo y, a la inversa, al papel de la sociedad en la traducción. Como historiador y crítico de la actividad versora, puedo especificar, aplicando criterios esencialistas, la validez lingüística que tiene la traducción de Il Cortigiano de Castiglione, realizada por el dúo Boscán/Garcilaso: si es correcta o no, si es adecuada o no. Pero, trascendiendo el texto terminal y no quedándome en él, puedo y debo 
comprobar si, además, el texto traducido ha provocado un efecto estilístico al imitar en español el efecto elegante del italiano original o si, como dijo Ambrosio de Morales en su Discurso sobre la lengua castellana, el Cortesano de Baldassarre Castiglione habló en España tan bien como en Italia, donde nació; podré y deberé investigar también si el ideal del hombre humanista propuesto en il Cortegiano tuvo un ef ecto social en el círculo de amigos que rodeaba a Carlos V en Toledo en el que, por cierto, moriría, víctima del cólera, Castiglione. $\mathrm{Si}$, por ejemplo, se comprobara que, llevado de los ideales «cortesanos» del libro, el duque de Alba hubiera puesto en práctica sus principios en el gobierno de los Países Bajos, se estaría manifestando una vez más que una traducción, en este caso la de la obra de Castiglione, leída por Álvarez de Toledo, quizás fue un elemento más en la conducción moral y política de una persona que tuvo repercusiones en todo un pueblo y en la época histórica que vivió. La averiguación de estos y muchos otros puntos será objeto de unos estudios de la traducción orientados sobre criterios humanísticos. La sociología de la traducción se nos da fundamentalmente en su historia. Y sin sociología poco nos interesa su esencia.

Una historia de la traducción así entendida viene a poner de cabeza, como decía Marx que él pretendía hacer con el sistema hegeliano, toda esta teoría hipertrofiada que desde Mounin acá nos ha llovido en medio siglo, el que va de 1950 a 2000 . La historia de la traducción considera lo que es la actividad, la teoría lo que debe ser pero quizás no es. Como símbolos ejemplares de estas dos actitudes científicas, la esencialista y la histórica, podrían hacerse valer los gestos que los dos grandes filósofos, Platón y Aristóteles, muestran en el célebre cuadro de la escuela de Atenas de Leonardo. El primero señala a las alturas celestes del concepto (el topos ouranou); el segundo, a la inmediatez de lo terrestre.

Ina historiografía legítima de la traducción deberá orientarse:

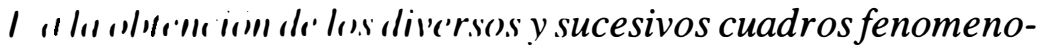

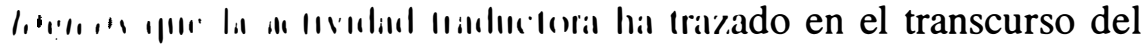


tiempo, integrando todos los factores determinantes y estableciendo términos/conceptos fácilmente utilizables en el manejo de la disciplina, como pueden ser, por ejemplo, la «traducción humanista», la «traducción durante la Reforma», las «bellas infieles», la traducción «abbasida», categorías útiles que, a costa de prescindir de matizaciones, hagan ganar unidad conceptual a los fenómenos estudiados. Para ello deberá implicar en la investigación todos los elementos que, como causa, motivo, condición o efecto explicativo, han intervenido en la fenomenología de la traducción a lo largo de la historia, tales como:

- El contexto económico, social y cultural en que se realiza. Desde este punto de vista, la historia de la traducción depende del conocimiento que le aporten la historia de las ideas, la sociología -público receptor, grado de cultura del mismo, afición por la lectura del mismo-, y la historia política del pueblo/lengua a la que se traduce.

- Igualmente habrá que considerar la intervención de agentes externos, como el papel de las agencias literarias, las ayudas estatales, de fuera o de dentro, etc. en el surgimiento de la traducción. Las ferias del libro suponen un impulso decisivo para el intercambio de material bibliográfico y, consiguientemente, al fomento intrínseco de la traductografía desde los postulados de la calidad y la comercialización. No desde la llamada sociedad civil, sino desde el poder político le advendrá a la traducción una promoción complementaria, pero importante, en la forma de los premios nacionales e internacionales de la traducción.

- La valoración del efecto que la actividad traductora ha conseguido en el cuadro histórico de su época, de su contexto, es decir, el múltiple efecto - social, cultural, literario—-de la traducción en el público destinatario. La traducción de un obra como la Summa Theologica de Santo Tomás de Aquino, ¿qué duda cabe que no tendrá el mismo efecto, la misma crítica en los años 
cincuenta de España, cuando existía una cultura católica que en el momento presente, en el que se ha consumado ya un proceso de laicización irreversible en la cultura española y en la que esa obra a lo sumo podrá ser objeto de curiosidad científica, filosófica, etc.?

- El elenco de traductores que marcan ese cuadro de época y figuran con derecho propio, por el número, la calidad o la eficacia de sus traducciones en la historia de la traducción ${ }^{12}$. La postulada transparencia del traductor ha inducido en alguna ocasión a construir historias de la traducción, monográficas o generales, dedicadas mayormente a la actividad traductora de los grandes escritores que, en ocasiones pro pane lucrandum, en ocasiones como ejercicio estilístico o, finalmente, comoalternativa en períodos de sequía espiritual, ejercen de traductores. Una auténtica historia de la traducción debería tratar, a la inversa, de rescatar, del anonimato y para la memoria, a toda esa cohorte de artesanos de la cultura literaria que cumplen con su función de mediar entre los pueblos y las lenguas. Se trataría de primar la historia de los traductores en el contexto de la historia de la traducción —en la que también podían entrar, por supuesto, los

$12 \quad$ Aquí resulta perentorio hacer una advertencia: en la historia de la traducción no vale esa simpática figura del musical americano Chicago, que hacía gala de transparencia. No vale jugar al "Mister Cellophan". El traductor nunca debe ser, socialmente, transparente. Marcada tendencia de nuestra época ha sido la reducción al anonimato del versor. Son muchas las publicaciones y editoriales que prescinden de la mención del traductor. Espasa-Calpe, meritoria desde muchos puntos de vista, no en último lugar por su elenco de cientos de títulos extranjeros de su Colección Austral, ha pasado en ocasiones del nombre de sus traductores, que con harta frecuencia no aparecen o lo hacen en «letra pequeña", la que no se lee. Lo mismo sucedió con la editorial Diana de Madrid o la editorial Bruguera de Barcelona. Frente la magnitud que ya en la década de 1950 adquiere la actividad editorial en el mundo y, correlativamente, la traducción, el traductor pierde opacidad y tanto el editor como el lector parecen pasar por alto la figura y el hacer del versor. Solo los autores que ejercen ocasionalmente como traductores, que son muchos, se utilizan como reclamo y figuran en portadas y folletos. En el caso del teatro, donde se somete las traducciones entregadas por los traductores a adaptaciones, la situación es aún más grave. En este contexto de depreciación de la labor traductora — Ortega llegó a llamar apocados a los traductores—, hay que inscribir la renuncia a los prólogos que anteriormente obligaba al traductor a exponer el cómo y el porqué de su versión: el aspecto biográfico de su realizador: procedencia preparación cultural y filológica, experiencia literaria, etc. 
autores-traductores-, lo que supondría hacer sujetos de esa historia toda una serie de nombres condenados al olvido y prescindir del carácter culturalmente clasista de la historiografía. Como traducción que funciona como texto propio en la lengua de destino quizás pueda hacerse valer con mención honorífica la traducción que Cernuda hizo de Hölderlin, aunque tan digno será el trabajo del anónimo traductor que rescata del olvido la aportación científica del biólogo o del matemático. Pero si en la historia de la traducción prescindimos del criterio de la excelencia y del criterio estético, es decir, lo que Lawrence Venuti llama la estética de la fluidez (fluency), la que pretende leer el texto original como si hubiera sido producido en la lengua terminal; si pretendemos que la historia de la traducción sea la historia de la comunicación de los escritos eficaz y realmente existente, habrá que salir de esa actitud de dulía, de servidumbre a los grandes literatos que, además de escribir, han traducido o adaptado. Otro caso sería el de los traductores que ocasionalmente se dedican a la escritura, en cualquiera de sus formatos: ensayos, poesía, etc. Hora es ya de que la historia de la literatura dedique su espacio al género traducción.

Así, por ejemplo, quien deseara trazar la historia de la traducción en la Cuba de Castro, sus realizaciones y deficiencias, más que reseñar los datos editoriales, que por lo demás estarán recogidos en los elencos de la UnEsCo, sin duda tendría que construir cuadros y períodos haciendo prevalecer en ellos causas, condicionamientos - también políticos - y efectos de la actividad traductora, atendiendo momentos tan concretos como el nivel de dirigismo político en la actividad editora de la isla, la penuria, políticamente motivada, de papel en el sistema castrista, los canales de distribución editorial; el grado de adaptación del libro a los postulados del régimen, el grado de divergencia que el traductor/ editor ha querido/podido manifestar con relación a la norma, etc., y a la inversa, el grado de efecto que esas 
traducciones han podido tener en la modificación de esa sociedad tan peculiar.

2. a la determinación de los marcos generales de estética traductiva, fijando en qué medida los componentes funcionales, estilísticos y estructurales de los textos originales se repiten en los textos terminales y si de estos resultan, primero, una traducción adecuada o inadecuada con relación al gusto de la época y, segundo, una corriente de pensamiento traductivo que haya determinado el modus operandi del traductor. Al igual que se han identificado ya pensamientos de estética traductora coherentes -el caso de las «bellas infieles» francesas o en el «fidelismo» ilustrado alemán-, ¿podrían construirse otros cuadros de pensamiento que pudieran figurar como realidades históricas?

$$
* * *
$$

Son estos, pues, los retos que actualmente tiene la historia de la traducción: hacer una historiografía integrada en la que se destaque la causalidad recíproca de versión y realidad social y cultural; en la que se calibre la medida en que la primera ha contribuido al progreso de los tiempos y las sociedades. Si, como dice George Steiner, «toda cultura es una serie de traducciones», la historia de la traducción está en el núcleo de toda analítica cultural. Tal es la tarea que un historiador de la traducción debe realizar: manifestar que su cultura es una serie de traducciones. 